\title{
Task analysis based on observing hands and objects by vision
}

\author{
Yoshihiro SATO Keni Bernardin ${ }^{\dagger}$ Hiroshi KIMURA Katsushi IKEUCHI ${ }^{\ddagger}$ \\ Univ. of Electro-Communications ${ }^{\dagger}$ Univ. of Karlsruhe ${ }^{\ddagger}$ Univ. of Tokyo
}

\begin{abstract}
In order to transmit, share and store human knowledge, it is important for a robot to be able to acquire task models and skills by observing human actions. Since vision plays an important role for observation, we propose a technique for measuring the position and posture of objects and hands in 3-dimensional space at high speed and with high precision by vision. Next, we show a framework for analysis and description of a task by using an object's functions as elements.
\end{abstract}

\section{Introduction}

It is important for a robot to be able to acquire task models and skills by observing human actions in order to transmit, share and store human knowledge. The strategy for understanding a human's action is the subject which should be tackled in order to solve processes such as studying and gaining experience from a human. Although research on teaching from observation is advanced on various points[1][2][3], the technique is still far from being applicable to real world problems, because of insufficient capabilities in observing and describing the real world. Since the aim of this research is the acquisition of human handworking skills, we tackle the problem of measuring the shape of a hand or an object's posture in 3-dimensional space with sufficient accuracy to do task analysis by vision. Furthermore, we tackle the problem of automatically generating a task description from the acquired vision information as an operation sequence on an abstraction level which can be understood by a human.

A human determines the optimal shape of his hand based on the task[4]. The used grasp form is useful for estimating the purpose of the task or of a specific state of the task. Moreover, the kinds of tasks a robot can execute can be increased by generating the robot's grasp operation automatically from observation of the human's grasp operation[5]. Many techniques have been proposed for recognizing the form of the hand, such as classification by silhouette, using devices such as sensor gloves, the eigen-space method by using depth images, etc. However, it is not desirable to use equipment which has physical restrictions like a sensor glove in a scene where a human and a robot do cooperative work. Recognition from arbitrary 3-dimensional positions is required, without being limited to specific viewpoints. Moreover, in conventional research, only the hand region was observed using a low resolution range sensor, and it was not intended to make a wide range observation of the task[6]. In this paper, in order to solve these problems, we perform hand shape recognition with the eigen space method by using a depth image from a high resolution range sensor, in which the hand region was clipped and normalized.

The principal subject of task analysis is to observe the state of objects, such as a tool, parts, etc. which are operated by a human. In order to analyze various operations, it is necessary to recognize objects with free curved surfaces, and to acquire their position and posture in 3-dimensional space continuously. The active search method, based on color histograms, is one of the techniques which look for a specific object in an image[7]. Using color histogram search makes the technique robust to little changes in form, and allows to find objects at high speed. However, it is difficult to look for a 3 -dimensional object whose silhouette changes with its posture, and it is necessary to use a previously created image database of all silhouettes to obtain a solution. In this paper, we propose a technique for high-speed search by generating the color histogram of arbitrary postures using a color model and a geometric model of the object. Furthermore, the object position and its posture are estimated by the ICP method using a depth image[9].

Task analysis expresses the state of the environment such as a human's action and tools from observation of the human's demonstration. The problem is how to find a good modelling framework, that is, how to design a description language for a recognition strategy. Since handwork is a task that relates to the mutual interaction of humans and objects, it is difficult to generally design the modelling framework. At this stage, various trials are still being performed. In this 


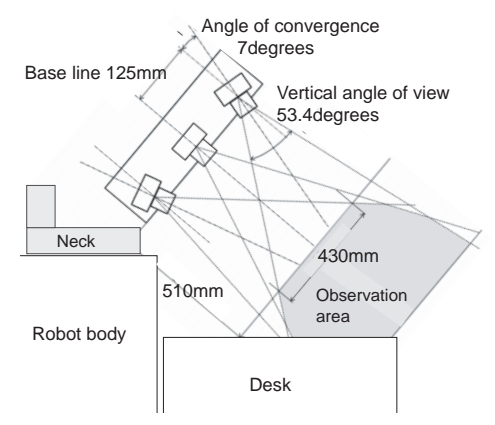

Figure 1: Observation environment

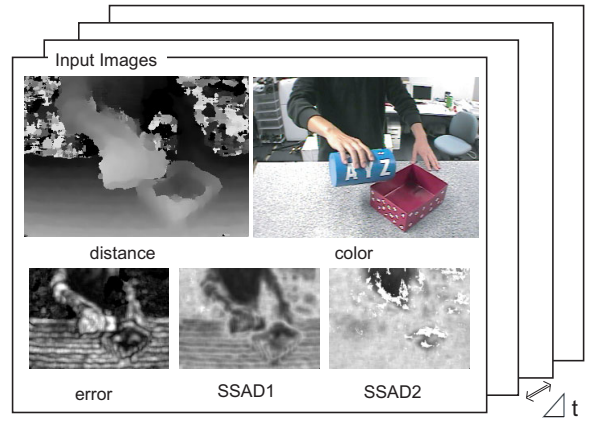

(a)

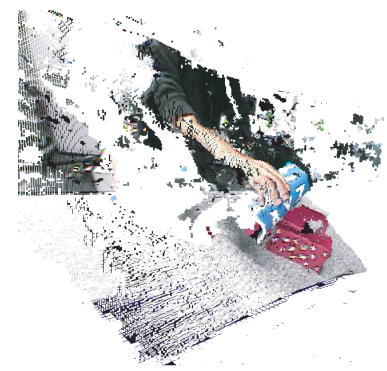

(b)

Figure 2: Input images: (a)Input image array (b)Side view of the distance image

paper, we concentrate on the problem of segmenting events from vision information. Information at a low degree of abstraction is an advantageous element for the flexibility of a model. The functional part of an object is treated as attention point in space, and the speed as attention point in time. We show examples of event recognition by evaluating distance and posture between the functional parts of objects.

\section{Vision Process}

\subsection{Observation Environment}

Here, we show the observation environment, composition, and input images. A side-view of the observation environment is shown in the Fig.1. Handwork performed on the desk set at a robot's front is observed by the range sensor located on the robot's head. The observable range is between about $500 \mathrm{~mm}$ - $1000 \mathrm{~mm}$ from the camera center. Since the resolution of the depth direction is 8 bits, precision is about $2 \mathrm{~mm}$. The size of an input image is $280 \times 200$ pixels. The input image sets are depth, color, SSAD, error, and sequence in time. The range sensor has a zoom function of 1.5 times to 2.0 times, obtained by using the electronic zoom function of the separate cameras(Fig.2(a)). The depth image obtained is the so-called 2.5-dimensional figure.(Fig.2(b)) The framerate is 30 frame/sec.

\subsection{Hand Shape Recognition}

In this section, we discuss the problem of recognizing the shape of a human's hand using the eigen space method on the distance and color images acquired by a high resolution range sensor.

Hand Shape Recognition using the Eigen Space Method. Although the eigen space method is known to be used to recognize object postures, estimate their position, etc. we use it to classify hand shapes and estimate hand positions. Generally the problem is the need to use normalized data as the input of an eigen space method. The process flow of hand shape recognition is shown in Fig.3. First, skin color is extracted from a color image and the region of the human's hand is clipped. The position of a 2dimensional image of the hand region is specified by template matching based on normalized correlation to the hand region of the last scene. The image of the obtained hand region is passed in an area filter, and noise is removed. The center of gravity of the resulting image of the hand region is acquired as the position of the hand at that time. The hand region image is normalized, including the depth direction, using the size in real space already obtained from the range sensor.

The depth value is treated as a vector and recognition performed using the eigen space method as follows:

1. The eigen vector of the covariance matrix of the vector group from study images is calculated.

2. The eigen space is constituted on the basis of the eigen vector with the largest eigen value.

3. Observation images and study images are projected and expressed as points in eigen space.

4. The distance between a pair of corresponding points is regarded as an evaluation measure for class resemblance.

Consequently, the hand shape in the current scene is acquired from the distance values for each class. For more robust estimation, the evaluation is done continuously in time. The hand shape is obtained from the minimum value of the distance values to the second power from a number of scenes in the past. 


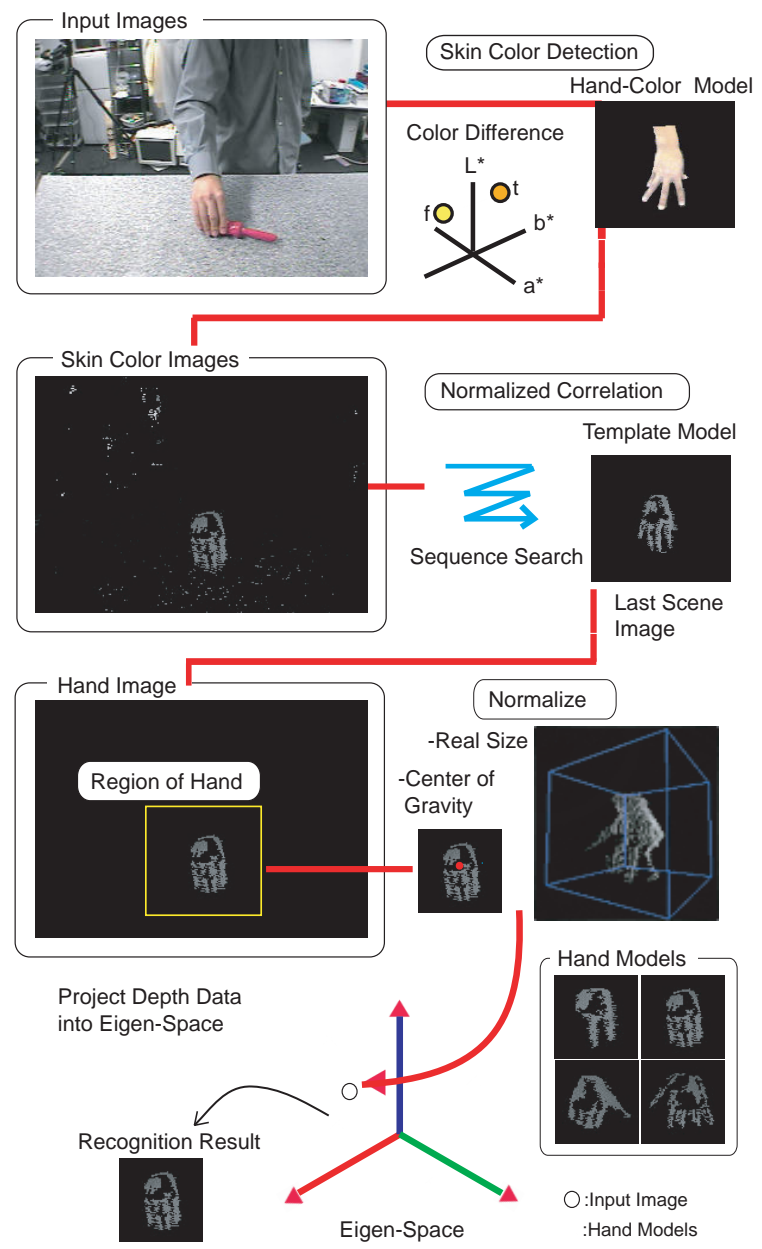

Figure 3: Hand-tracking sequence

\subsection{Object Tracking}

In this section, we discuss a method for 3dimensional object tracking combining object region search in the 2-dimensional image by color and recognition of the 3-dimensional position and posture of the object by the ICP method.

Tracking of the object region by color classification. Generally, searching the object region in a 2-dimensional image by color offers the advantage that one can obtain the edge of an object region clearly and at high speed, and the drawback that recognition of a 3 -dimensional object is difficult. On the other hand, the ICP method can estimate the posture of an object with complicated form, but it needs exact range data. However, with the stereo vision system based on area matching used in this research, no clear range data of the edges of objects is obtained. In this paper, we argue that in combin-

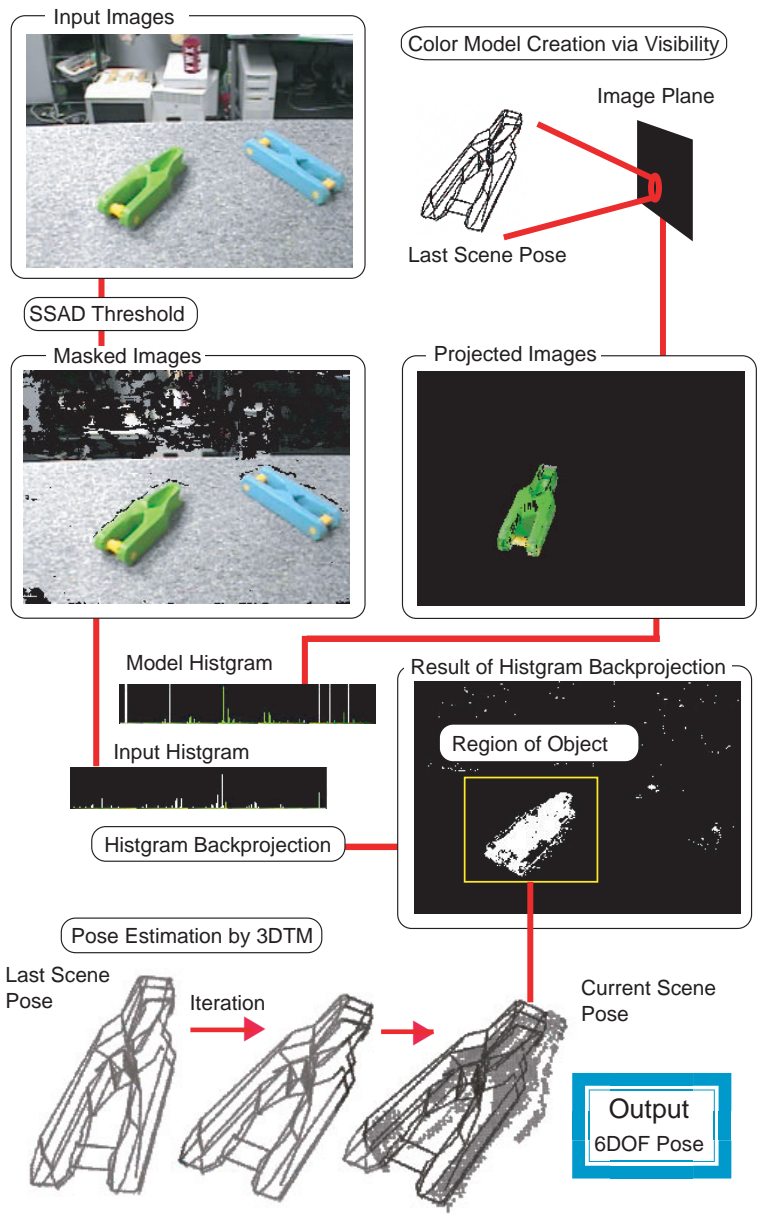

Figure 4: Object-tracking sequence

ing the two techniques, they compensate each other's faults.

The process flow of object region tracking by color is shown in Fig.4. Color histograms are generated from an observation image and the silhouette of an object model, respectively, and the object region is extracted by comparison.

The vote box $I[r][g][b]$, a 3-dimensional array which divides into $Q_{c}$ onst pieces each of the 3-dimensions of color space for storing a color histogram, is prepared. The color histogram of an observation image is generated by scanning the pixels one by one, and voting a corresponding color to one corresponding vote box. Next, the color histogram of an object model is generated. Object information is given beforehand as a geometric model and a color model. The objects, such as a tool, are usually colored by two or more colors. Since there are differences in the views of an object depending on its posture, the color 
of the mesh of the last observed scene is used to draw a model image. Like the color histogram of the observation image, the 3 -dimensional vote box for the object model histogram is prepared, a projection image of the object's posture is scanned pixel by pixel, and the color histogram of the model is generated.

Next, the degree of similarity of the two histogram is checked. The ratio $R[r][g][b]$ of the color histogram of an object model and an observation image is calculated, and normalized so that its size is in the range $0-1$.

$$
R_{[r][g][b]}=\min \left(\frac{M_{[r][g][b]}}{I_{[r][g][b]}}, 1\right)
$$

If an observation image is replaced with the value of this ratio, a region with a color close to the object's color will take a value nearer to 1 , and the object region will be extracted. Labeling of the object region is carried out, and region with values below a threshold are removed. The depth image is masked by the pixels which remained and an output in form of 3-dimensional point group data is obtained.

Estimation of object's posture by the ICP method. The 6DOF position and posture are obtained by fitting the 3 -dimensional point group data and the point of an object geometry model's mesh. In this paper, the ICP method known as 3-d template matching is used. Since this method uses Mestimators for optimization of a posture, it handles well the noise in the observation image and the incorrect correspondence between the object model and the observation image. The estimation of the object posture can be performed based on the following four procedures.

1. Visible points of the object model are evaluated from the normal direction of the meshes of the object model and the viewpoint.

2. The closest correspondence between visible points of an object model and the dots of the object region in the observation image is found.

3. An initial position and posture are estimated using the least-squares method.

4. An optimization is performed by repeated correction so that the distance between corresponding points becomes small, using a statistical Mestimator technique.

In the repetition operation of the ICP method, it happens that the object position and posture do not converge, but oscillate. To remedy this, a kalman filter is applied. Moreover, an object speed can also be obtained from the state value estimated by the kalman filter, and used for the task analysis. This is shown in the following chapter.

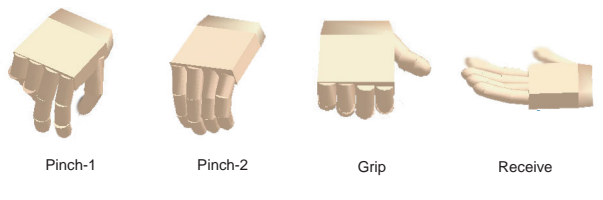

Figure 5: Hand classes

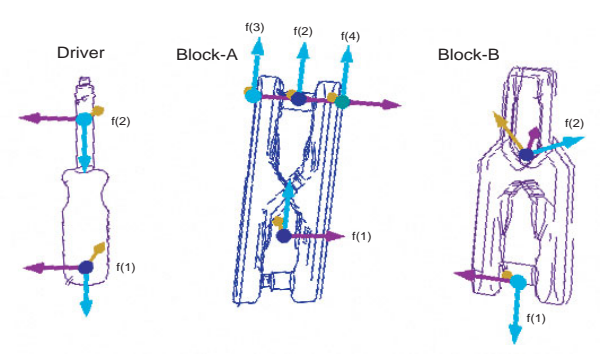

Figure 6: Objects functions

\section{Task Analysis}

In this section, we explain task analysis on an example in part assembly. The analysis is based on the segmentation of events by paying attention to object speed and position.

\subsection{Primitives for the task analysis}

We defined four hand shape classes that will always be well visible at the time of work. The Eigen space was generated using study images of 25 pose variations of each hand shape. Here, the eigen space of a right hand and left hand was prepared, and both hands have been recognized.

The object for observation is shown in Fig.6. The point which should be noted is defined as a functional part on the object's geometric form. The object's functional part such as a hole or an axis is expressed by a coordinate system on the object. It was manually determined a priori.

The event to recognize is defined as the state of an operation, expressed as relations between functional parts of two objects, and the kind of operation is described as a predicate. For example, the event in which the right hand holds the object Obj's functional part 1 is described: "Right-Hand Grasped Obj.f [1]". The objects and operations which are used as observation primitives in this paper are arranged in Table3.1. An example of the contents of a task is shown in Fig.7. There is the task of attaching Block-A and Block-B, and of them fixing using a 


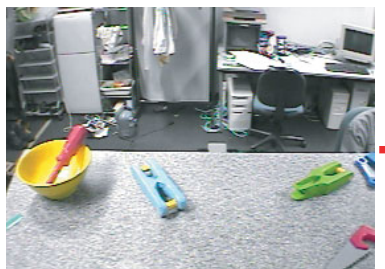

(1) Initial Scene

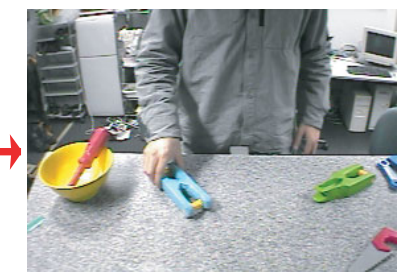

(2) Right-Hand Grasped Block-A

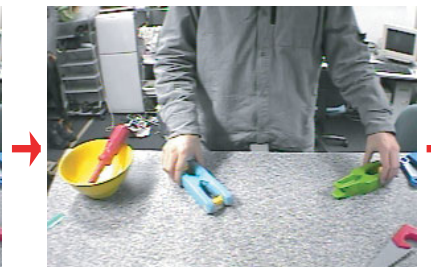

(3) Left-Hand Grasped Block-B

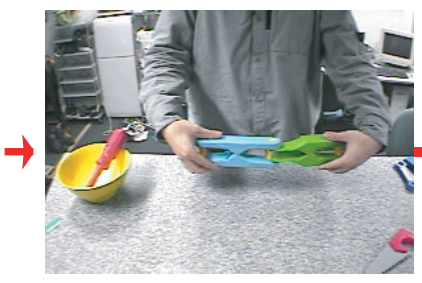

(4) Block-A \& Block-B Fixed

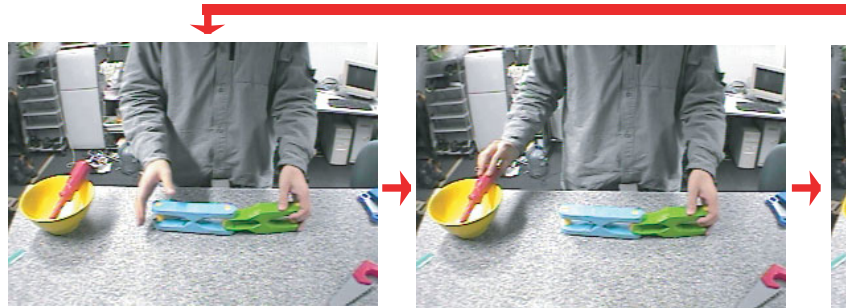

(5) Right-Hand Released Block-A

(6) Right-Hand Grasped Driver

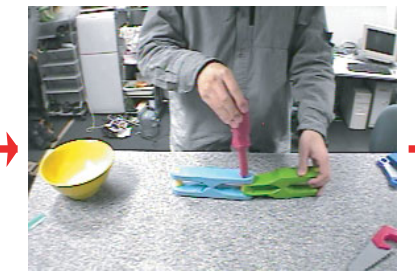

(7) Block-AB \& Driver Fixed

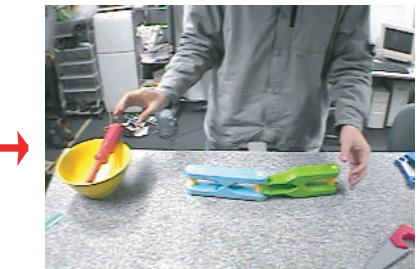

(8) Right-Hand Released Driver

Figure 7: Observation results

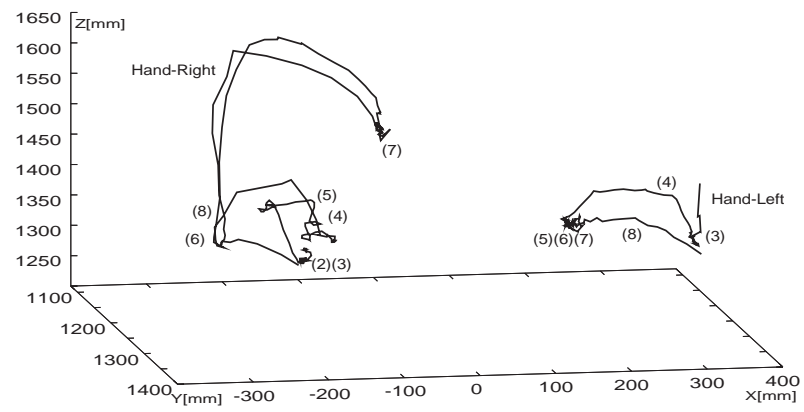

Figure 8: Trajectory of hands

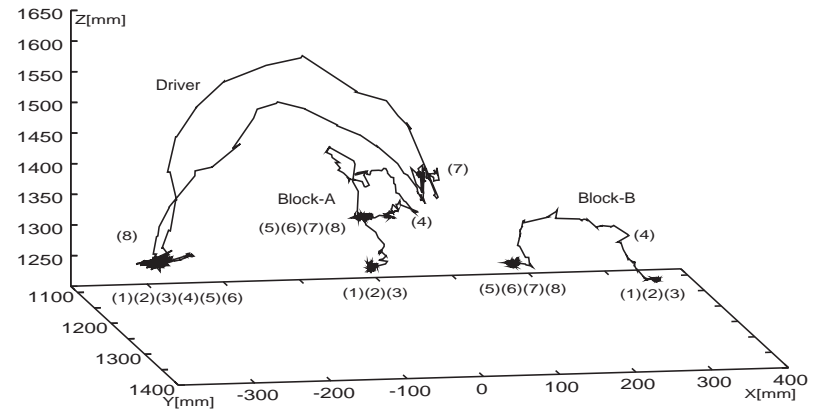

Figure 9: Trajectory of objects
Screwdriver. The trajectories of hands and objects obtained from vision are shown in Fig.8 and Fig.9.

Table3-1 Observation elements

\begin{tabular}{|c|c|c|c|}
\hline object & $\begin{array}{l}\text { Right-Hand } \\
\text { Left-Hand } \\
\text { Block-A } \\
\text { Block-B } \\
\text { Driver }\end{array}$ & operation & $\begin{array}{l}\text { pinch-1 } \\
\text { pinch-2 } \\
\text { grip } \\
\text { receive } \\
\text { release } \\
\text { fix }\end{array}$ \\
\hline
\end{tabular}

\subsection{Event detection by speed of an object's functional part}

If we assume that only man's hand and the object move, observation will focus on the thing which is moving. Since people have a tendency to perform operations involving object contact slowly, carefully, speed can be used as feature to detect an event. The model controlling attention for event detection is defined by making the speed of a functional part of an object into a parameter.
States of attention are "disregard", "caution", and "gaze." The allowed change of states is "disregard" "caution" "gaze" "disregard". The "gaze" state is defined as a trigger for event detection by geometric evaluation. When an object's functional part moves from a still state to a state of operation, the state it changed from "disregard" to "caution". Event detection is triggered when shifting from operation to stillness, i.e. the state is changed from "caution" to "gaze." If the system stays in the "gaze" state for a while, it will shift to the "disregard" state.

\subsection{Event detection by position of object's functional parts}

The event detection by spatial evaluation is performed as follows: The distances between each functional part of objects whose positions were expressed within the world coordinate system are calculated. The position of the center-of-gravity and the finger- 


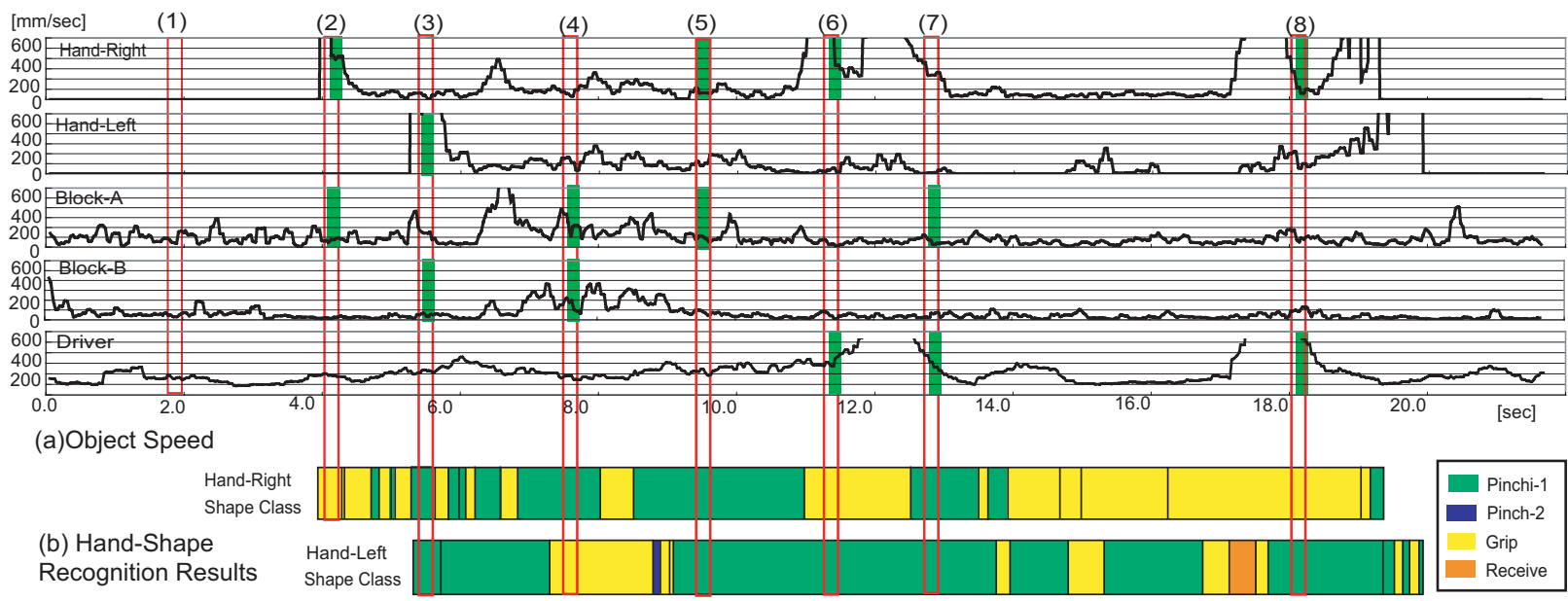

Figure 10: Analysis of observation results

tips of the human's hand in a world coordinate system are calculated. The functional part in the "gaze" state and other functional parts are scanned one by one. The relation between two functional parts is evaluated based on distance and posture, and it is judged as follows whether an event happened or not: When the distance between functional parts is less than a threshold value, contact is recognized. Furthermore, when the posture of the coordinate system of two functional parts is in agreement, it is recognized that axis alignment was performed. The method of grasping operation of the human's hand on the object is described by the shape of the human hand, such as Pitch, Grip, etc.

\subsection{The task analysis experiment}

The result of task analysis based on the speed of objects and hand shape is shown in Fig.10. The detected events were able to be described as follows with the time they occured at marked in parentheses. (1)Initial scene, (2)Right-Hand Grasped BlockA.f[1], (3)Left-Hand Grasped Block-B.f[1], (4)BlockA.f[2] Block-B.f[2] Fixed, (5)Right-Hand Released Block-A.f[1], (6)Right-Hand Grasped Driver.f[1], (7)Driver.f[2] Block-A.f[3] Fixed, (8)Right-Hand Released Driver.f[1].

\section{Summary}

This paper showed a method for tracking the position and posture of objects by vision in real space and real time using a high-speed range sensor of high resolution, and also showed a method for recognizing hand shapes. Moreover, we showed a framework for task analysis and showed an experimental result for the example of assembly work on objects.

\section{Acknowledgments}

This work is supported in part by the Japan Science and Technology Corporation (JST) under the Ikeuchi CREST project, and in part by the Grant-in-Aid for Scientific Research on Priority Areas (C) 13224025 of the Ministry of Education, Culture, Sports, Science and Technology.

\section{References}

[1] K.Ikeuchi,T.Suehiro: Toward an Assembly Plan from Observation Part I: Task Recognition With Polyhedral Objects, IEEE Trans. Robotics and Automation,Vol.10,No.3,pp.368-384,1994.

[2] Y.Kuniyoshi,M.Inaba,H.Inoue: Learning by Watching: Extracting Reusable Task Knowledge from Visual Observation of Human Performance,IEEE Trans. Robotics and Automation, Vol.10,No.6,1994.

[3] R.Zollener,O.Rogalla,J.M.Zollner,R.Dillman: Dynamic Grasp Recognition within the Framework of Programming by Demonstration,Int. Conf. of Roman,2001.

[4] M.R.Cutkosky,R.D.How: Human grasp choice and robotic grasp analysis,Dextrous Robot Hands,SpringerVerlag,pp.5-31,1990.

[5] S.B.Kang,K.Ikeuchi: Grasp Recognition and Manipulative Motion Characterization from Human Hand Motion Sequences,Proc. of ICRA94,pp.1759-1764,1994.

[6] H.Kimura,T.Horiuchi: Task-Model Based Human Robot Cooperation Using Vision, Proc. of IROS1999,pp.701706,1999

[7] M.J.Swain,D.H.Ballard: Color Indexing,Int. Journal of Computer Vision,vol7,pp11-32,1991.

[8] T.Kanade,H.Kano,S.Kimura,E.Kawamura,A.Yoshida,K.Oda: Development of a Video-Rate Stereo Machine, Journal of the Robotics Society of Japan, Vol.15,No.2,pp.99105,1997

[9] K.Kawamura,K.Hasegawa,Y.Someya,Y.Sato,K.Ikeuchi: Robust Localization for 3D Object Recognition Using Local EGI and 3D Template Matching with MEstimators,Proc. of ICRA2000,Vol.2,1848-1855,2000. 